\title{
"Littérature d'aveu" et prise en charge des valeurs dans Ennemis publics de Michel Houellebecq et Bernard-Henri Lévy
}

Assuming Values in "Confession Literature": Ennemis publics by Michel

Houellebecq and Bernard-Henri Lévy

\section{Roselyne Koren}

\section{(2) OpenEdition Journals}

\section{Édition électronique}

URL : http://journals.openedition.org/aad/1002

DOI : 10.4000/aad.1002

ISSN : 1565-8961

\section{Éditeur}

Université de Tel-Aviv

\section{Référence électronique}

Roselyne Koren, "Littérature d'aveu" et prise en charge des valeurs dans Ennemis publics de Michel Houellebecq et Bernard-Henri Lévy ", Argumentation et Analyse du Discours [En ligne], 5 | 2010, mis en ligne le 20 octobre 2010, consulté le 23 septembre 2019. URL : http://journals.openedition.org/aad/ 1002 ; DOI : 10.4000/aad.1002

Ce document a été généré automatiquement le 23 septembre 2019.

\section{(c)}

Argumentation \& analyse du discours est mis à disposition selon les termes de la licence Creative Commons Attribution - Pas d'Utilisation Commerciale - Pas de Modification 4.0 International. 


\title{
"Littérature d'aveu" et prise en charge des valeurs dans Ennemis publics de Michel Houellebecq et Bernard-Henri Lévy
}

\author{
Assuming Values in "Confession Literature": Ennemis publics by Michel \\ Houellebecq and Bernard-Henri Lévy
}

Roselyne Koren

En octobre 2008 paraît, en co-édition Flammarion-Grasset, un volume de correspondance intitulé Ennemis publics, co-signé par Bernard-Henri Lévy et Michel Houellebecq ${ }^{1}$. Cet ouvrage ne donne pourtant pas à lire des "lettres ordinaires » définies comme des messages qui «parlent de choses et d'autres (aussi quotidiennes et banales que possible), sans avoir d'enjeu capital»(Kerbrat-Orecchioni 1998: 15). Il s'agit au contraire de lettres qui sondent les choix existentiels effectués par leurs auteurs. Sans doute le livre ne se limite-t-il pas à cette quête " des vraies questions »: c'est un ouvrage de combat contre les « meutes » de journalistes, de critiques littéraires ou de contemporains en tous genres qui les ont poursuivis ou les poursuivent encore de leur «haine », et c'est dans cette optique qu'il commence par un "coup d'envoi » ironique qui simule l'auto-flagellation: "Nous sommes l'un comme l'autre des individus assez méprisables", "A nous deux, nous symbolisons parfaitement l'effroyable avachissement de la culture et de l'intelligence françaises » (2008:7-8). Audelà, cependant, de cette polémique à quatre mains, cette correspondance comporte des enjeux éthiques étroitement liés aux pratiques de la «littérature d'aveu», en l'occurrence au parler vrai introspectif indissociable d'un questionnement sur « le moi profond » opposé au « moi social ».

2 On tentera de justifier l'hypothèse qu'à travers le genre épistolaire, les deux auteurs tentent de se soustraire aux diabolisations médiatiques et de substituer à un espace discursif "fermé » (Maingueneau 1998: 71) par ces images caricaturales, un espace interactionnel ouvert en perpétuel devenir. Il ne s'agit pas pour eux de rédiger une 
"grande entreprise autobiographique " à la manière de Rousseau, mais d'« expérimenter ", dans le cadre d'un genre qui n'est ni philosophique, ni littéraire, ni médiatique, les tenants et aboutissants de l'énonciation de "vérités autobiographiques » (2008: 47). Le choix du genre épistolaire est donc étroitement lié ici à la décision de se livrer, dans le cadre d'un "travail de parole" (306), à un questionnement éthique existentiel où les deux auteurs exploreront ensemble leur rapport au parler vrai introspectif et à la justification de leurs croyances axiologiques respectives. Ce questionnement comprend une réflexion et un débat sur le malaise suscité par les normes préétablies du sens commun, la primauté de l'autonomie du sujet, le rôle joué par l'Autre, la fonction primordiale de la prise en charge de la vérité, mais aussi d'autres valeurs comme le juste et le bien et le besoin de transcendance du sujet. Celui-ci oscillerait entre une "éthique de conviction" et une "éthique de responsabilité $»^{2}$ qui régulent son rapport à l'inaction ou à l'action, soit à l'engagement civil ou politique ou au non-engagement.

On suivra donc ici les étapes suivantes: 1. reconstitution du métadiscours des deux auteurs sur les liens qui lient le genre épistolaire au parler vrai et au parler juste ; 2 . analyse du "travail de parole » consacré à la notion de «littérature d'aveu ", soit à la problématisation de la question de la "vérité » et de la véridiction du métadiscours sur le «moi profond»; 3. passage de la prise en charge du vrai à celle d'autres valeurs motivant le choix de l'action ou du refus d'agir dans la vie politique et sociale. On insistera constamment, chemin faisant, car la rhétorique des lettres et la primauté accordée par les deux auteurs au pouvoir des mots $(251,253-256,273-274,300)$ nous y invitent, sur la contribution active de figures de rhétorique à la mise en mots épistolaire de la justification de soi.

\section{Genre épistolaire et contrat de véridiction dans Ennemis publics}

4 Essayons d'abord de reconstituer, à partir de remarques disséminées dans la trame de l'ouvrage, le discours des deux auteurs sur la contrainte de rectitude aléthique inhérente au genre épistolaire. BHL et Houellebecq se disent décidés à respecter les closes d'un contrat de véridiction et de sincérité; la confiance réciproque des correspondants serait à ce prix. Houellebecq rappelle ainsi dans sa lettre du 8 février 2008 (33) que "Schopenhauer note avec surprise qu'il est relativement difficile de mentir par lettres "; il ne peut, poursuit-il, qu'abonder dans son sens et "noter »à son tour "avec surprise» qu'il y a quelque chose dans l'échange épistolaire qui «vous pousse à la vérité, à la présence». La lettre aurait «une force authentifiante immédiate " (63). D'où la nécessité, quand on a décidé de "faire un bout de chemin, ensemble » (78), de ne pas se contenter de donner les motivations superficielles de ses actes et d'en poursuivre la justification en mettant en mots les "vraies » causes, les raisons les plus profondes, les plus secrètes ou les plus difficiles à avouer ${ }^{3}$. Les gratifications ne se font pas attendre: on est en droit d'attendre de l'autre qu'il vous croie sur parole, même s'il est sous le coup d'une "espèce de choc " (28) dû à des explications jugées inintelligibles. Et si «malentendu » il y a, on n'aura de cesse qu'il ne soit « dissipé » (156). Il peut certes y avoir des moments d'" insincérité », conscients et délibérés ou non. Ils seront désignés et explicités avant de mettre un terme à la correspondance, non pas au nom d'un soudain accès de repentance, notion de morale 
dénuée de sens pour les deux auteurs, mais par respect de l'engagement envers une des règles les plus fortes du jeu épistolaire: la véridiction (324). Alors qu'un discours monogéré permet à l'énonciateur soit de respecter les règles du parler vrai, soit de recourir au mensonge ou à la fiction, le genre épistolaire impliquerait par contre, du fait de sa dynamique interactive fondationnelle, des normes aléthiques incontournables. Comme il est linguistiquement impossible de voir si le contrat a réellement été respecté, si les récits autobiographiques sont sincères et quel est le degré de confiance qui règne entre les deux auteurs : on s'en tiendra à l'analyse de ce qui est visible et interprétable, à savoir les déclarations des deux auteurs et leurs prises de position axiologiques.

5 La littérature d'aveu est déclenchée, stimulée et orientée par les mots de l'Autre. Il ne s'agit donc pas d'une pratique discursive monogérée, même si chacun des auteurs est libre de choisir les thèmes ou les mots qu'il prendra en ligne de compte, n'a pas à réagir à chaud et dispose du temps nécessaire à la réflexion ${ }^{4}$. L'enjeu des débats n'est pas la persuasion. Houellebecq ne dément pas la déclaration de BHL qui affirme ne pas croire aux vertus argumentatives du dialogue ni surtout à «la théorie » affirmant qu'il "suffirait de s'opposer, de confronter des arguments et des contre-arguments pour que se dissipent, par enchantement, les ténèbres de l'ignorance : les gens, dans la plupart des discussions arrivent avec leurs convictions ; ils repartent avec les mêmes » (306). La visée de l'échange est, d'un commun accord, une meilleure compréhension de la «vision du monde » de l'Autre, cet étranger que la correspondance va partiellement transformer en intime (7-8, 251, 306-309). Les conditions interactionnelles d'un questionnement éthique heuristique sont ainsi réunies. Les deux correspondants vont se pousser réciproquement « à fouiller dans le tas de secrets » (187) à la recherche de la « logique profonde » de leurs actes (256). Il s'agit de creuser sous la surface, d'« en venir au fond » (166), de " se mettre au clair » avec certaines questions cruciales comme celle de l'engagement (76), bref, de se livrer à un exercice d'introspection stimulé par les mots de l'autre, ces mots qui donnent «l'envie d'embrayer » (51) dès que celui-ci vous les a «tendus » (59). Il s'agit donc de tirer profit d'interactions verbales placées sous le signe de la sincérité et de la rectitude, interactions qui aboutissent à un sentiment de « libération» (314).

6 Les débats épistolaires d'Ennemis publics ne problématisent pas la décision de publier la correspondance. Ce silence est à première vue paradoxal : les deux auteurs insistent en effet sur leur méfiance à l'égard de la «littérature d'aveu » qui a le dévoilement du «moi profond" pour objet. Ils reconnaissent leur décision d'arborer en public des masques qui les protègent contre les attaques des journalistes. Or, les débats contiennent de nombreux récits autobiographiques traitant de questions aussi intimes que les relations des auteurs avec leur père et leur mère, leur rapport à la religion, leur vie sexuelle... C'est donc ici le silence sur les motivations de la publication qui semble lourd de sens et doit être problématisé. On peut avancer trois hypothèses explicatives. 1) La publication de la correspondance permet de discréditer les portraits diffamatoires diffusés par la «meute» des ennemis en tous genres sans avoir à leur reconnaître le moindre statut d'interlocuteur. Le blason du «moi social » sera ainsi redoré aux yeux des futurs lecteurs de l'échange épistolaire qui défend de "vraies valeurs contre les vains parleurs » (Maingueneau 1998:69);2) l'échange épistolaire s'adresse, par-dessus leurs têtes, à tout lecteur que l'on qualifiera ici, avec Maingueneau (1998:62), de «destinataire indirect» ou d'« Autre ultime» (ibid. : 70) invité ainsi à "gloser» les 
textes sans avoir à subir la pression des médias ${ }^{5}$ - cet « Autre » aura à interpréter et à évaluer les diverses options offertes par un questionnement éthique sur le sens de l'existence, l'essence de l'homme, la prise en charge du vrai et celle des valeurs qui conduisent à l'action ou au refus d'agir ; 3) les lettres échangées par Houellebecq et BHL comprennent des débats jugés d'intérêt public par les deux auteurs dans le sens où elles "visent à participer directement à un débat public existant ou à en ouvrir un " (Maingueneau 1998: 58). Le questionnement interactionnel auquel se livrent les deux auteurs participe en effet à des interrogations à la fois anciennes et toujours actuelles, comme ce qui fait sens pour l'homme et pour l'écrivain, ou comme les tenants et aboutissants de l'action dans la société civile.

\section{La vérité épistolaire dans tous ses états}

On essaiera à présent de recenser et d'interpréter les divers types de vérités évoqués dans la trame de ces échanges épistolaires. Ce qui compte en l'occurrence, soit «la question des questions ", « la question, la vraie » (40), c'est tout d'abord le travail de parole des deux auteurs. Celui-ci est comparable à une descente spéléologique dans les profondeurs du moi où serait ancrée la «logique profonde de nos actes» (256). On ne peut espérer y accéder que si l'on creuse à force de mots sous la surface du «moi social ", à la recherche d' "histoires personnelles», de leurs "dénis", d'" effrois subjectifs », de «blessures mal guéries » constituant le « roman familial, pour le coup, inavoué " (40-41). Parler vrai, c'est alors reconnaître que la "clef " serait ainsi pour chacun des deux auteurs le «mode d'être et de vie » de leur père (40-42, 49-51, 53-56, 174-175). Le récit autobiographique agit sur l'ensemble de l'échange auquel il confère un halo d'authenticité. Occuper une "position d'énonciateur qui se qualifie comme individu doué d'une biographie et d'une image singulière » (Maingueneau 1998:69) et non comme intellectuel médiatique, écrivain célèbre dont l'identité se résumerait à celle de son moi social, apparaît ici comme un mode d'humanisation et donc de valorisation de l'ethos des deux auteurs. Parler vrai, c'est encore renoncer à se livrer une fois de plus à des "raisonnements trop carrés pour être honnêtes » (40) et se montrer tel qu'on est, avec ses contradictions et ses zones d'ombre. La vérité sur «le moi profond", ce "minotaure tapi au fond de son labyrinthe de mots» (39), peut consister alors dans le fait d'admettre qu'il est le plus souvent indicible (35-39) ou que chaque "aveu " ajoute "une couche de mystère " (45). Nous sommes donc bien ici, pour le meilleur et pour le pire, dans le fief de la « littérature de l'aveu» ${ }^{6}$, soit du récit autobiographique, type de discours dont le contraire serait, affirme Houellebecq, « la littérature de l'imaginaire » (33).

Ultime avatar de cette exploration des voies menant aux vérités autobiographiques : la dynamique interactionnelle inhérente au genre épistolaire, dynamique qui décentre l'écriture et la fait passer de l'introspection individuelle à une aventure heuristique

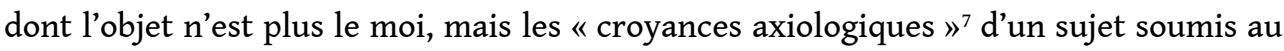
regard critique et aux questions de l'Autre. Les débats épistolaires ont alors pour fin de permettre moins de "savoir qui l'on est», que de découvrir ce qui compte véritablement pour chacun, soit les valeurs fondamentales qui président aux choix existentiels et, comme on le verra plus loin, à une conception de l'engagement dans la vie sociale. 
On analysera en guise d'illustration l'un des débats les plus véhéments qui les oppose l'un à l'autre et qui a pour objet la défense du positivisme par Houellebecq et sa mise en cause par BHL. Il s'agit en l'occurrence de se positionner par rapport au statut de la vérité référentielle. L'affrontement est déclenché par le contenu d'une remarque apparemment anodine de BHL à propos d'une cause de mésentente antérieure. Celle-ci concerne le choix par Houellebecq du comparant métaphorique "pierre " afin de désigner la nature du sujet, choix épicurien matérialiste, énergiquement contesté par $\mathrm{BHL}^{8}$. Celui-ci introduit la présentation et la justification de son point de vue en ces termes : « Alors image pour image, et tant qu'à faire, je vais vous en proposer une autre. Je ne dis pas qu'elle soit meilleure. Ni, naturellement, plus "vraie" (comme si c'était la question!)» (134). Houellebecq voit dans le contenu de cet énoncé entre parenthèses qui conteste la primauté absolue de la vérité référentielle un symptôme inquiétant. Il n'existe pas à ses yeux de valeur supérieure à la vérité; il affirme au contraire que "c'est précisément la question » et que «l'honneur tout particulier de la philosophie occidentale est d'avoir mis au premier plan la question de la vérité, jusqu'à tout lui sacrifier $»^{9}$. Nietzsche, poursuit-il, aurait été le premier à tenter d'« entacher de soupçon la quête de la vérité, et, nommément, de la vérité scientifique. Il a ainsi inauguré en philosophie ce qu'on pourrait appeler l'ère de la déloyauté» (151). Laisser glisser la "question de la vérité » " au second plan », ce serait en fait " en revenir aux sophistes » (ibid.). Houellebecq se présente donc comme un "positiviste endurci » (154) qui ne croit que dans les affirmations attestables et réfutables de réalités prouvées par « la méthode des sciences » (152). Il reconnaît que cette " conception déspiritualisée » (ibid.) de l'éthique le conduit à refuser «l'engagement» (153) pour s'en tenir à une éthique de conviction objectiviste.

10 Ceci explique le rôle qu'occupe la description (150) dans ses argumentaires. L'une des illustrations les plus frappantes de ce choix rhétorique est la description de paysages irlandais (Houellebecq réside alors en Irlande) qui sert d'entrée en matière à la réponse du 10 avril 2008 (142). Houellebecq y réagit en effet à une lettre où BHL a présenté et justifié des choix éthiques existentiels radicalement différents des siens. Le fait que sa réaction à ce positionnement "métaphysique " commence par une description de paysage ne saurait être interprété comme une simple technique de référence à la situation d'énonciation. Sans doute pourrait-on se contenter d'y voir, dans les termes de Kerbrat-Orecchioni (1998: 17), l'évocation du «cadre spatio-temporel dans lequel s'inscrit son activité d'écriture » en raison de la «situation non partagée » du moment de rédaction de la lettre. Cette interprétation, certes légitime, n'est cependant pas la seule possible dans ce contexte. La description manifeste également le refus de l'ancrage dans une "métaphysique " "spiritualiste ", le refus de toute forme de transcendance autre que la référence à ce que l'auteur considère comme le summum du vrai : les réalités empiriques extérieures au sujet, réalités observables et irréfutables. Parler vrai dans une telle perspective, c'est refléter les réalités extralinguistiques « telles qu'elles sont " et considérer l'explication comme une procédure "objective" spéculaire, conforme aux "principes de la preuve» (150), car celle-ci est l'un des «seuls principes » «qui n'auront jamais trahi l'homme dans sa recherche de la vérité » (151). Ceci implique le rejet des «métaphysiques diverses, matérialistes et spiritualistes, qui se partagent le marché de la croyance » (150).

11 Il n'est donc pas étonnant que la métaphore du miroir utilisée par Houellebecq afin de définir les normes rhétoriques de son écriture joue un rôle central dans l'élaboration 
des positions respectives des épistoliers quant au statut de la vérité. Cette métaphore lexicalisée est l'un des emblèmes d'une conception réaliste objectiviste de la sémantique ${ }^{10}$ qui relègue dans le non-dit le raisonnement suivant: le langage est une somme neutre d'instruments techniques qui ne font pas écran entre la pensée et les choses; il peut donc dire des vérités factuelles préétablies qu'il se contente de refléter. Houellebecq recourt à ce trope dans l'une des dernières lettres de l'ouvrage (295-296) et BHL réfère à cette «théorie du miroir » dans la lettre suivante (308-309). Le romancier positiviste est conscient des limites de cette métaphore : il en joue tandis qu'il évoque le fait que "les miroirs sont déformants", que "certains de plus sont encrassés, tavelés » et ne peuvent donc plus "refléter grand-chose » (296). Il redoute même que ne vienne le jour où le miroir de ses dires «viendra à se fendiller, puis à éclater en fragments " (ibid.), mais il n'en continue pas moins à penser l'acte de dire en termes spéculaires et donc vériconditionnels. BHL substitue une image radicalement différente à celle du miroir et donc une autre "théorie » de l'interprétation qui défend une conception très différente du rôle du langage. Celui-ci n'y est pas présenté comme une somme technique d'instruments à vocation descriptiviste, mais comme le lieu fondamental où se pratiquent la réflexion et l'interrogation sur le sens du monde et de l'existence. Les «Commentaires », affirme-t-il, « ne sont pas les reflets mais les piliers d'un monde, qui, sans cela, retournerait au néant; les livres sont, non le miroir, mais les poutres de l'univers» (308-309). BHL ne donne donc pas la primauté au pouvoir aléthique représentationnel du langage, mais à un pouvoir critique d'analyse et d'interprétation qui constitue avant tout à ses yeux le propre de l'homme et sa raison d'être.

\section{Action et engagement au prisme des valeurs}

Nous avons vu que Houellebecq présente la question de ses réserves quant à l'engagement dans la vie publique comme la conséquence directe de sa décision de donner la primauté au vrai sur le juste (153): il ne pourrait y avoir engagement ni action, à ses yeux, car seules comptent des vérités incompatibles avec la vie politique et sociale $\mathrm{du}$ fait de leur caractère absolu et irréfutable. BHL défend une thèse radicalement inverse qui a la prise en charge de valeurs autres que la vérité ou l'évidence empirique absolues pour objet. Deux conceptions de l'éthique s'opposent donc ici : l'une prône l'inaction au nom d'une conception absolutiste de la primauté du Vrai et l'autre, l'action au nom de croyances axiologiques d'ordre "métaphysique». Nous consacrerons donc ce développement à la confrontation des tenants et aboutissants de deux visions du monde antithétiques. La première ne jure que par ce qui est passivement accepté tel quel, tandis que l'autre s'interroge sur un devoir-être qui transcende les faits avérés et aspire à trouver dans l'action l'assouvissement de désirs de "dépassement" (81). L'apologie de l'action ou de l'inaction à laquelle se livrent respectivement BHL et Houellebecq a, tout d'abord, leur conception de la nature du sujet pour fondement.

On peut certes considérer que les valeurs qui régissent nos choix et nos actes sont ancrées a priori dans des systèmes imposés de l'extérieur par le sens commun et par les contraintes inhérentes aux discours sociaux circulant dans les espaces publics, mais si l'on croit dans l'autonomie, même partielle, du sujet d'énonciation, il est nécessaire de s'interroger d'entrée de jeu sur la conception du sujet prônée par les deux auteurs. On 
choisira comme exemple type un débat où Houellebecq et BHL s'affrontent, comme dans le cas de la "théorie du miroir", par métaphores interposées. Houellebecq assimile ainsi les êtres humains, dans une lettre du 24 mars 2010, à des «pierres, lancées dans le vide, et aussi libres qu'elles; ou, si l'on tient absolument à voir les choses du bon côté ", à "des comètes " (123). BHL s'empare de ce comparant et le soumet à une critique systématique qui passe par deux étapes : a) critique de l'image du corps inanimé plongé dans le vide qu'il relie sur un mode analogique à d'autres types de corps sans vie, « lancés dans le vide ", soit « gouffre » où " les pierres se cognent [...] les unes contre les autres", "guigue des cadavres des suppliciés dans les cercles de l'Enfer de Dante ou «chute des corps du 11 septembre qui se sont jetés du haut des tours » $(130) ; b$ ) évocation du " côté sans retour » de la chute et du discours de Lucrèce qui insiste dans le De natura rerum sur le fait "qu'on ne peut plus faire autrement " alors que de "descendre et de descendre encore » (ibid.). Ces commentaires traduisent un sentiment de malaise qui déclenche des jugements de valeur négatifs. L'image des corps lancés dans le vide et la théorie de Lucrèce ne peuvent expliquer, selon BHL, du fait de leur matérialisme, "l'apparition de ce caillou qu'on appelle une conscience et dont chacun sent bien qu'il est difficilement réductible à une somme de particules, surgies de rien" (131). Et quand bien même on supposerait, "par on ne sait quelle acrobatie mentale» qu'une addition de cailloux puisse aboutir un jour à «la constitution d'une âme ", ce ne pourrait être, affirme BHL, du fait de la métaphore de la pierre, qu' « une âme pétrifiée, ossifiée, formée une fois pour toutes, lisse comme un galet, monolithique, sans devenir, sans faille " (132). Or, le sujet est, à ses yeux, une instance en perpétuel devenir $(288,314)$. Les qualifications concrètes subordonnées cidessus au mot "âme» transforment l'assimilation initiale à une "pierre " en une métaphore filée dont toutes les composantes impliquent des jugements de valeur. Ceux-ci distinguent entre ce qui est présenté comme le Mal - le figement, la réduction résiduelle d'un corps à sa plus simple expression, l'évolution impossible, l'uniformité absolue - et inversement le Bien, soit les notions inverses de vitalité, de créativité, de devenir, de changement et de pluralité ouverte. Le « devenir-sujet » que défend BHL est une procédure dynamique où le moi est en gestation perpétuelle au contact des autres sujets, mais aussi du fait des multiples situations auxquelles il est amené à se mesurer ; il ne peut être désigné que par des vocables comme « rendez-vous d'identités multiples, brisées, contradictoires " $(132,39)$. BHL va donc remplacer la thèse épicurienne de Houellebecq par ce qui est à ses yeux «le grand récit alternatif»: celui de la Bible à laquelle il emprunte la métaphore du « vent » ou encore "souffle » divin insufflé dans l'homme, soit ruah en hébreu.

Ce comparant, qui réfère à des qualités comme le mouvement et l'élan qui stimulent le dépassement du malaise, est donc substitué à celui de "pierre » par rapport auquel il présenterait, affirme BHL, six avantages majeurs. Nous n'évoquerons ici que les trois derniers car ils concernent la possibilité du sujet, la forme du sujet et l'intersubjectivité (134-140). Ce sujet, BHL le considère comme conscient, libre et foncièrement différent de «ce sujet fatal, cette petite sphère toute ronde, cernée une fois pour toutes, détachée du monde extérieur, que nous raconte la théorie du caillou » (137). La notion de sujet ne renverrait donc pas à un état, mais à un procès (138), procès d'individuation complété et structuré par l'intersubjectivité dont l'épicurisme ne pourrait rendre compte. "Il y aura autant de passerelles que de gouffres, de mondes partagés que de mondes en guerre ou en débat». Le sujet de BHL dément le "sentiment de solitude qu'imposent les philosophies atomistes ", il pratique une éthique de l'interaction qui 
" arrache le sujet à un égoïsme qui, sinon, ferait de sa singularité [...] une prison ou un linceul» (139). La plupart des vocables utilisés ici véhiculent des évaluations axiologiques implicites négatives ("gouffres", "solitude", " égoïsme", "prison", "linceul»). Ces évaluations sont toutefois positives dans le cas de "passerelles", « monde partagés », « singularité ». BHL remplace donc l'extériorité que constituent les réalités objectives chères au sujet matérialiste, par l'extériorité de l'Autre par rapport à l'Un ${ }^{11}$, cet Un que Levinas considère, rappelle BHL (140), comme «l'otage » et " 'l'obligé' d'autrui ». Il brosse ainsi, face à l'ethos du positiviste qui prône le refus de l'engagement au nom d'une conception absolutiste et donc inaccessible de la Vérité, l'ethos d'un " intellectuel "engagé" » qui a besoin pour exister de "se sentir comptable de l'autre " (141) et pour qui la solidarité et l'action sont motivées par la recherche métaphysique du vrai, mais aussi avant tout du juste et du bien.

On passera à présent de ces deux conceptions antithétiques du sujet à l'analyse du débat des deux auteurs sur la question de l'engagement. La démarche suivie par BHL a pour enjeu la justification des raisons profondes de son choix de l'action dans la vie politique et sociale. Son argumentaire passe délibérément des motivations les plus superficielles à celles qu'il considère comme les plus pertinentes et les plus authentiques. Il commence donc sa descente dans les profondeurs de la logique de ses actes par la désignation des motivations apparentes dont il lui arrive de jouer dans les espaces médiatiques, soit le " numéro de bien-pensant, redresseur de torts idéologiques et rappeleur à l'ordre des bons sentiments " (74). Sincérité, véridiction et rectitude obligent. La vraie question, souligne-t-il dans un second temps, est cependant

ce qu'on a dans la tête quand on décide, ou qu'on feint de décider, que le sort du genre humain vous indiffère - ou quand on endosse le rôle, à l'inverse, de celui qui [...] choisit de faire comme si le malheur des autres le concernait, comme s'il en était comptable et même un peu responsable et comme si l'on était vraiment « homme » que si l'on se sent, au moins pour partie, chargé des autres hommes et leur otage (75).

Les « vraies » causes ${ }^{12}$ de l'engagement de BHL sont au nombre de trois et classées par ordre croissant d'importance: «le goût de l'aventure» qui implique « une autre relation à la mort donc à la vie, à la peur donc au sentiment d'exister » (79), « le goût de la performance ", cette tentation "de faire ce que les autres ne font pas ou de le faire, s'ils le font, d'une façon qui n'appartient qu'à moi » (79), et le "goût de vivre au-dessus de soi » (78-82). Cette construction qui va de la réfutation initiale des motivations apparentes aux motivations les plus fortes et les seules qui puissent être jugées totalement authentiques, est spécifique d'une logique des valeurs entendue comme logique de l'évaluation du vrai et du préférable, et non pas de l'assertion ou de l'acceptation absolue de l'évidence. Cette logique distingue ici entre le vrai et le faux, l'apparence et la réalité, mais aussi entre le bien et le mal, le juste et l'injuste face à cet autre, Houellebecq, son correspondant, mais aussi face au lecteur anonyme dont il se sait probablement aussi l'« otage ».

17 Le genre épistolaire, on l'a vu, implique un contrat de véridiction et de sincérité. Deux des trois dernières motivations (le goût de l'aventure et de la performance) sont donc présentées comme des défauts qu'il tient à reconnaître et à qualifier en recourant aux épithètes subjectives axiologiques suivantes : "indécentes, obscènes, m'as-tu-vu, déplacées » (79). La troisième et dernière motivation de son goût pour l'engagement est assurément la plus forte et la plus éloquente. L'idée d'un désir de "dépassement de soi » (81) et l'aspiration à «se hisser [...] au dessus de soi, de sa taille et du destin qui 
vous a été donné » correspond exactement au sens que Polin $(1977: 48-49,55)$ donne aux termes de "dépassement spirituel » et de "transcendance qui nous servent de critère dans la détermination des valeurs ». Contrairement à Houellebecq qui croit dans la transcendance d'une valeur de vérité existant indépendamment de l'homme et de ses actions et reflétée par le miroir des mots et de la pensée, BHL considère comme Polin (1977 : 59) que le transcendant doit être considéré comme « un élan de création ou de dépassement » ancré dans le sujet. Si l'on veut « expliquer la présence de valeurs pour l'homme ", poursuit Polin, on est conduit «à le doter lui-même de cette puissance de transcendance par laquelle il pourrait se dépasser et se créer. Les valeurs en seraient la première manifestation » (ibid.). Ce besoin de transcendance ne saurait être assimilé à la foi qui anime le croyant. BHL se déclare athée (156). Son argumentaire confirme par ailleurs la pertinence de la remarque de Danblon (2005: 95) sur la compatibilité d'une rationalité « certes laïcisée, mais qui n'a pas renoncé pour autant à un ancestral besoin de sacré ». On peut considérer que ce besoin de dépassement de ses propres limites ou de celles imposées par un système de valeurs préétabli, extérieur au sujet et jugé insuffisant ou inadéquat, « correspond peut-être tout simplement à ce que l'on nomme l'éthique » (ibid. : 91).

18 La prise de position de Houellebecq est exactement inverse, il excelle ici dans la fonction d'anti-modèle. Il s'agit pour lui de justifier un total désintérêt pour toute forme d'engagement. Dans une lettre qui répond précisément à celle de BHL commentée ci-dessus, la structure même des "aveux» de Houellebecq suit un ordre contraire à celui suivi par BHL puisqu'au lieu de suivre un ordre croissant qui place la justification la plus forte, la plus valide et la plus valorisante à la fin, elle suit un ordre décroissant qui passe des « raisons honorables » aux « raisons douteuses, et de pire en pire » afin d'expliquer pourquoi il n'est pas, et ne souhaite pas, être un intellectuel engagé (85). Les motivations successives de son non-engagement sont « une modestie idéologique confinant à l'athéisme » (87), le refus de se considérer comme un "citoyen » (88) et la préférence argumentée pour une identité « dépolitisée » (90) qui le conduit à préférer le statut d'" usager ", classé par ses soins dans la catégorie de notions à valeur négative référant à un type d'appartenance sociale «qui s'effondre ", «s'avilit» (91). Houellebecq considère enfin comme "les raisons non-honorables» de son nonengagement (ibid.), « la renonciation à la violence physique comme méthode principale de règlement des conflits ", l'incapacité viscérale « d'obéir ", la " lâcheté » qui consiste à renoncer à « une opposition frontale » et, en cas de guerre, le choix du camp de « ceux qu'indiffère le destin de la démocratie, de la France libre, de la Tchétchénie ou du Pays basque » (92-93). Parler vrai oblige, Houellebecq affiche sans le moindre masquage un " parti pris d'égoïsme et de lâcheté » (93) qui le conduit à parler «en pratique » «au nom d'à peu près rien » (ibid.), exception faite du cas de la violence physique en temps de guerre qui conduit « des guignols sanglants à s'emparer de n'importe quel prétexte, idéologique ou religieux " pour détruire les viaducs, chemins de fer, immeubles de bureaux que d'honnêtes techniciens ont construit (94).

19 Le parti pris réaliste et matérialiste affiché par Houellebecq se situe ici à rebours des prises de position axiologiques de BHL et de celles du sens commun : il condamne certes la cruauté ou la folie des "guignols sanglants ", mais pas afin de rendre justice à leurs victimes; ce qui le conduit à renoncer ponctuellement et très partiellement au nonengagement, c'est la destruction du fruit technique de leurs travaux. Ce raisonnement paradoxal pourrait être considéré comme métonymique. On pourrait formuler l'hypothèse que les viaducs, immeubles, bureaux détruits renvoient aux hommes qui les 
ont construits, mais cette hypothèse perd sa pertinence face aux assertions suivantes : "Je manifeste une certaine compassion à l'égard du malheureux, mais elle est très peu différente de celle que j'ai devant un animal pris au piège ", "quant à la notion de dignité humaine, j'avoue que je n'y comprends absolument rien » (153).

Il existe cependant à côté de ces multiples énoncés d'auto-dénigrement un passage contradictoire (mais on a vu plus haut que parler vrai pouvait référer à la désignation des contradictions $\mathrm{du}$ « moi profond») qui complexifie l'auto-portrait du romancier car il abonde ponctuellement, à son corps défendant, dans le sens de la thèse du dépassement défendue par BHL. La sincérité de l'autocritique conduit Houellebecq à se demander, alors que l'échange épistolaire touche à sa fin, s'il « serait peut-être temps " qu'il « prononce lui aussi ses adieux à la raison. Raison qui ne lui a servi à rien, qui ne lui a jamais permis de produire une ligne, qui n'aura fait toute sa vie que le tourmenter par le caractère désespéré de ses conclusions " (272). Ce questionnement s'engage même davantage encore sur la voie d'une rationalité axiologique puisque l'on trouve sous la plume de Houellebecq, à quatre reprises consécutives, des énoncés qui font état d'incertitudes et de désirs d'auto-dépassement concernant le rapport entre science et philosophie, soit vérité positiviste et questionnement métaphysique, et la conviction qu'il n'existe aucune logique d'ordre supérieur dans le monde. Le quatrième et dernier énoncé : «Comme j'ai du mal, en un mot, à me passer d'une mystique » (273) - confirme que le besoin ancestral de sacré évoqué par Danblon (2005) n'est pas totalement étranger à Houellebecq en dépit de son positivisme radical ou peut-être précisément à cause du caractère par trop absolu de ce positivisme. On peut reconnaître dans l'énoncé anaphorique «j'ai du mal à » et dans les passages des vers d'Aragon que le romancier cite aussitôt après -

Je ne sais ce qui me possède

Et me pousse à dire à voix haute

Ni pour la pitié ni pour l'aide,

$\mathrm{Ni}$ comme on avouerait ses fautes,

Ce qui m'habite et qui m'obsède

- un désir subreptice de transcendance, dont l'énonciation à voix haute est rendue possible par la littérature de l'aveu. Houellebecq se fait ici brièvement l'écho d'une aspiration plus ou moins consciente et assumée («je ne sais ce qui me possède », « me pousse à dire ", «ce qui m'habite et qui m'obsède »), aspiration qui prouve que ni la métaphore de la pierre ni celle du miroir ne peuvent rendre compte du rôle joué par les valeurs dans un questionnement éthique existentiel.

\section{Conclusion}

21 L'échange épistolaire publié dans Ennemis publics est certes à l'origine un échange de lettres personnelles. Les deux auteurs savent néanmoins dès le départ que cette correspondance sera publiée. L'enjeu de leurs débats n'est donc pas d'exprimer en privé des états d'âme, ni de jouir des plaisirs que l'on peut éprouver en profitant du «droit subjectif d'afficher ses opinions » (Meyer 2008 : 195). Il s'agit pour eux de sonder et de justifier des prises de position existentielles. Les voies axiologiques que suit la recherche des motivations de leurs choix respectifs confirment la pertinence de la conception des valeurs défendue par Meyer (ibid.) qui voit en elles des "passions désubjectivisées ». La perspective de la publication « institutionnalise » leur " travail de parole» et le transforme donc, comme le ferait, dans un échange de lettres 
personnelles, le passage de confidences intimes dénuées de distance critique à des jugements de valeur réciproques. On n'est plus alors dans le registre des questions de goût, mais dans celui des questions d'éthique qui créent les conditions de possibilité de «la discussion et de l'opposition» (ibid.). "Argumenter avec des valeurs, et pour des valeurs " exige, poursuit Meyer, une «élévation que méprise la jouissance passionnelle $(2008: 196)$.

e genre épistolaire n'est pas le seul espace discursif où des valeurs sont invoquées afin de justifier des croyances et des prises de position axiologiques. La rhétorique argumentative et les affrontements polémiques d'ordre politique et social leur accordent également une place notoire. Le récit aurait également, affirment les narratologues, un questionnement et des prises de position éthiques pour enjeux et, de fait, les deux auteurs d'Ennemis publics accordent une place primordiale dans leurs lettres au récit de l'histoire de leurs relations avec leurs parents. On essaiera donc au moment de conclure de confronter brièvement la contribution de l'échange épistolaire et de ces deux autres genres aux pratiques discursives de l'évaluation verbale à caractère axiologique.

L'argumentation a pour fin de persuader un auditoire, de susciter son adhésion et de l'inciter à l'action, mais elle peut aussi avoir la confrontation d'idées pour unique enjeu heuristique. Le désaccord est alors perçu comme un type de relation dialectique stimulant et bénéfique. L'échange épistolaire que l'on vient d'analyser est proche de ce second type d'argumentation: on peut y discuter et s'y justifier avec véhémence et passion ou dans une atmosphère dédramatisée de conversation et de confidence intimes, sans devoir contrecarrer des tentatives de persuasion. Seuls les lecteurs, interlocuteurs indirects de chacun des deux auteurs, peuvent être considérés du fait de la publication de la correspondance Houellebecq-BHL comme la cible de prises de position à visée persuasive. On est dans les deux cas - argumentation et échange épistolaire - dans une éthique du discours qui accorde à l'Autre une place primordiale : la rationalité et la validité des dires de l'Un dépendent du regard critique de l'Autre. Houellebecq reconnaît ainsi que BHL l'a contraint à s'avouer et à énoncer des vérités qu'il tentait jusque là d'occulter (299). L'autre n'est donc pas, comme dans de nombreux discours polémiques, une cible à détruire ou à convertir. L'argumentation peut, enfin. être privée ou publique, la lettre de même : mais seul le discours polémique a la prise de parole en public pour trait distinctif. La fin, de plus, peut y justifier les moyens : la rectitude et les vérités référentielles y comptent moins que la victoire et les chances de conversion des tiers à la cause défendue. Le débat épistolaire tel que les deux auteurs le pratiquent se distingue donc du discours polémique opposant les deux «ennemis publics" à leurs dénonciateurs par le contrat de véridiction, de confiance et de sincérité qui les lie l'un à l'autre. La mise en œuvre des valeurs ne se limite pas enfin dans cet échange, comme dans le cas de l'argumentation, à la prise en charge du vrai, elle inclut la prise en charge de valeurs comme le juste et le bien, la solidarité ou la compassion. La place accordée à des considérations d'ordre axiologique dans ces trois types de discours augmente toutefois la visibilité d'un trait distinctif transgénérique que l'on pourrait nommer avec BHL le dépassement de soi. On n'en est plus alors au stade des aveux, soit d'un dévoilement narcissique de secrets autobiographiques, dévoilement qui répugne aux deux auteurs ${ }^{13}$, mais à celui de la prise de conscience d'un aspect fondamental du questionnement éthique. 
Ce phénomène expliquerait selon Angenot (2008: 441-44) que les polémiqueurs continuent de combattre et de se justifier même si les échanges auxquels ils participent sont en fait des "dialogues de sourds" stériles. Ils y seraient poussés par un besoin d'auto-dépassement lié à une conception idéale de l'auditoire universel. L'aspiration humaine à la légitimation et à la validation des positions de l'Un par l'Autre serait d'autant plus forte que leur travail de parole s'effectue dans un univers imparfait où le vraisemblable occupe la place du vrai et le raisonnable et le préférable, celle de la raison absolue et des évidences établies a priori. Cette aspiration surgirait et jouerait un rôle décisif dès que l'enjeu de l'échange est la justification de croyances axiologiques et de choix existentiels par un énonciateur conscient du fait que sa recherche d'accords sans failles est condamnée à rester sisyphique. Dernier avantage et non des moindres de ce trait distinctif transgénérique pour le discours critique sur les valeurs: la confirmation de la thèse selon laquelle les croyances axiologiques ne sont pas uniquement ancrées dans des systèmes inhérents aux doxa et aux genres discursifs circulant dans les espaces publics. Elles sont aussi assumées par des sujets à la fois contraints et autonomes. Ceux-ci n'acceptent que ce qui fait sens pour eux et prouvent ainsi que la notion de rationalité axiologique n'est pas un vain mot.

\section{BIBLIOGRAPHIE}

Angenot, Marc. 2008. Dialogues de sourds (Paris : Mille et une nuits/Fayard)

Boudon, Raymond. 1995. Le juste et le vrai (Paris : Fayard)

Boudon, Raymond, 2009. La rationalité (Paris : PUF)

Danblon, Emmanuelle. 2005. La fonction persuasive (Paris : Colin)

Houellebecq, Michel \& Bernard-Henri Lévy. 2008. Ennemis publics (Paris : Flammarion Grasset)

Kerbrat-Orecchioni, Catherine. 1998. «L'interaction épistolaire », Siess, Jürgen (éd.). La lettre entre réel et fiction (Paris : SEDES), 15-36

Koren, Roselyne. 1997. « Le miroir virulent »,Scolia 10, « Rencontre linguistiques en pays rhénan $8 », 161-182$

Koren, Roselyne. 2008. " "Ethique de conviction" et/ou "éthique de responsabilité". Tenants et aboutissants du concept de responsabilité collective dans le discours de trois quotidiens nationaux français ", Questions de communication 13, 25-45

Koren, Roselyne. (à paraître) « La logique des valeurs selon Perelman et sa contribution aux linguistiques du discours »

Maingueneau, Dominique. 1998. « Scénographie épistolaire et débat public », Siess, Jürgen (éd.).

La lettre entre réel et fiction (Paris : SEDES), 55-71

Meyer, Michel. 2008. Principia Rhetorica (Paris : Fayard)

Polin, Raymond. 1977 [1944]. La création des valeurs (Paris : Vrin) 
Siess, Jürgen (éd.). 1998. La lettre entre réel et fiction (Paris : SEDES)

\section{NOTES}

1. Désormais BHL et Houellebecq.

2. Cf., au sujet des définitions et des enjeux de ces notions Koren 2008 : 29-34.

3. Cf., à ce sujet, p. 14, 240-241, 78-79.

4. Cf., à ce sujet, Kerbrat-Orecchioni 1998: 16-17, 35-36, Maingueneau 1998: 59 et BHL, p. 185-186.

5. Houellebecq souligne ainsi qu' «il a établi, au fil des années, avec ses lecteurs, une certaine relation de confiance (et que ces lecteurs inconnus ou non sont même, au monde, les seules personnes à l'egard de qui il se sente un devoir) ». Il " aurait l'impression, en trahissant cette confiance, de céder à la meute » (270-271).

6. Cf., entre autres, au sujet du métadiscours dont ce terme est l'objet dans l'ensemble du volume, les pages $35,45,47,75-76,97$.

7. J'emprunte cette dénomination à Boudon $2009: 79$.

8. BHL lui oppose la métaphore du «vent » ou «souffle » divin, spirituel (v. plus loin: 3. « Action et engagement au prisme des valeurs »).

9. Cf., au sujet de la primauté de la prise en charge de la vérité et des tenants et aboutissants de la critique perelmanienne de cette primauté, Koren :« La logique des valeurs selon Perelman et sa contribution aux linguistiques du discours ", à paraître.

10. Cf. Koren 1997 au sujet de cette métaphore emblématique.

11. Cf., au sujet de ces deux types d'extériorité antithètiques et de leurs conséquences quant à la définition de la notion d'objectivité Polin (1977: 19-20). Il y affirme, entre autres, que « l'objectivité est définie en termes d'extériorité » "à la pensée d'une personne donnée » dans le cas du rapport au réel extralinguistique, mais qu'il en est autrement lorsque ce qui est extérieur, c'est la personne de l'autre qui est à la fois «intériorité et extériorité », similarité et différence. «L'objectivité résulte » alors « de l'accord de soi-même et de l'autre » et non plus du reflet fidèle de l'objet dans les mots spéculaires d'un locuteur-miroir.

12. On a renoncé ici, faute de place, à l'analyse du recensement des pseudo-raisons ou des raisons considérées comme non-valides qui sont énumérées conformément au contrat de véridiction qui régule l'échange. Cf., à ce sujet, p. 76-77.

13. Cf., ainsi, ce passage de la lettre de BHL : «Et, quand je dis "le" moi, je ne pense évidemment pas à Sa Majesté le Moi avec son narcissisme, son miroir, son capital de ruses et de secrets » (p. 288).

\section{RÉSUMÉS}

L'échange épistolaire n'est pas le seul cadre discursif où l'on peut observer la façon dont des croyances axiologiques se trouvent justifiées. Quels avantages a-t-il pu présenter aux yeux des deux auteurs d'Ennemis publics, le philosophe Bernard-Henri Lévy et le romancier Michel Houellebecq? La prise en charge de vérités autobiographiques et la notion de «littérature d'aveu» occupent une place centrale parmi ces avantages: elles nous permettront de 
problématiser la question de la lettre, «laboratoire de valeurs». Le questionnement éthique auquel ils se livrent les incite ainsi à s'interroger sur leurs conceptions de la nature du sujet, de la responsabilité, de l'engagement et de l'action. On analysera, chemin faisant, quelques tropes spécifiques de leurs "visions du monde " antithétiques et on tentera de rendre compte du fait que leurs débats permettent de partager une aventure heuristique qui défige leurs images publiques respectives, objets de critiques virulentes de la part de nombreux journalistes et de commentateurs. L'évocation polémique de ces critiques joue certes un rôle important dans Ennemis publics, mais elle ne fera pas l'objet de cette contribution. On tentera ici de démontrer que cette correspondance met en œuvre et concrétise quelques-uns des concepts et des questionnements majeurs inhérents à la réflexion philosophique sur l'éthique.

The epistolary genre is not the only discursive framework in which the justification of axiological beliefs can be observed and analyzed. Asking why it was selected by the authors of Ennemis publics, philosopher Bernard-Henri Lévy and novelist Michel Houellebecq, will enable us to present different aspects of their respective attitude towards the accountability for autobiographical truths. The ethical issues in which they indulge allow them to confront their own conception of the nature of the subject, of responsibility, of engagement and action. We analyze some specific tropes of their antithetical world views, while trying to show how their debates have enabled them to share a heuristic adventure aimed at enhancing their public image, but discredited by a "bunch" of journalists and hostile critics. This is not, however, the main interest of this correspondence which displays and embodies some of the concepts and major concerns inherent in philosophical ethical thought.

\section{INDEX}

Keywords : epistolary, ethics, justification, logic of values, self

Mots-clés : genre épistolaire, justification, logique des valeurs, moi, questionnement éthique

\section{AUTEUR}

\section{ROSELYNE KOREN}

Université Bar-Ilan, ADARR 